\title{
Modelling the Interruption on HCI Using BDI Agents with the Fuzzy Perceptions Approach: An Interactive Museum Case Study in Mexico
}

\author{
Ricardo Rosales ${ }^{1, *(\mathbb{D})}$, Manuel Castañón-Puga ${ }^{2}\left(\mathbb{D}\right.$, Felipe Lara-Rosano ${ }^{3}$, Richard David Evans 4 (), \\ Nora Osuna-Millan ${ }^{1}$ and Maria Virginia Flores-Ortiz ${ }^{1}$ \\ 1 Accounting and Administration School, Autonomous University of Baja California, Tijuana 22390, Mexico; \\ nora.osuna@uabc.edu.mx (N.O.-M.); vflores@uabc.edu.mx (M.V.F.-O.) \\ 2 Chemistry and Engineering School, Autonomous University of Baja California, Tijuana 22390, Mexico; \\ puga@uabc.edu.mx \\ 3 Complexity Science Center, National Autonomous University of Mexico, Mexico 04510, Mexico; \\ flararosano@gmail.com \\ 4 Business Information Management and Operations Department, University of Westminster, \\ London NW1 5LS, UK; R.Evans@westminster.ac.uk \\ * Correspondence: ricardorosales@uabc.edu.mx; Tel.: +52-664-294-5884
}

Received: 27 July 2017; Accepted: 9 August 2017; Published: 13 August 2017

\begin{abstract}
Technological advancements have revolutionized the proliferation and availability of information to users, which has created more complex and intensive interactions between users and systems. The learning process of users is essential in the construction of new knowledge when pursuing improvements in user experience. In this paper, the interruption factor is considered in relation to interaction quality due to human-computer interaction $(\mathrm{HCI})$ being seen to affect the learning process. We present the results obtained from 500 users in an interactive museum in Tijuana, Mexico as a case study. We model the HCI of an interactive exhibition using belief-desire-intention (BDI) agents; we adapted the BDI architecture using the Type-2 fuzzy inference system to add perceptual human-like capabilities to agents, in order to describe the interaction and interruption factor on user experience. The resulting model allows us to describe content adaptation through the creation of a personalized interaction environment. We conclude that managing interruptions can enhance the $\mathrm{HCI}$, producing a positive learning process that influences user experience. A better interaction may be achieved if we offer the right kind of content, taking the interruptions experienced into consideration.
\end{abstract}

Keywords: human-computer interaction; ambient intelligence; interruption factor; belief-desireintention agents; perceptual computing; Type-2 fuzzy inference system

\section{Introduction}

We are currently involved in an information revolution where technology facilitates tasks and the daily activities of people, making them more productive; however, it is important to evaluate if the evolution of technology is truly helping people. In some cases, technology is not necessarily appropriately adopted; for example, if something that is new or novel is being misused, impacting misinformation and ignorance, it could mean the opposite [1]. In order to argue how technology can help in the interaction among educators and learners during the learning process, it is desirable that educators act as facilitators in the learning process, meaning that they are more interested in the learners learning than in the delivery of teaching. 


\subsection{The Learning Process}

The learning process is a relatively permanent change in an individual's behaviour, affecting their knowledge, attitudes, and skills, and can occur at any place or time consciously or subconsciously. In nearly all cases, the educator and learner are essential in the delivery of the process. They both build and play a leading role in teaching and learning. The learners gain more autonomy as they progress in the educational system, improving their interactions with the help of technology. It is important to also consider the educator as an identifying model; the learner is often influenced by the educator's different ways of thinking, speaking, and acting. The success of the learning process depends on effective collaboration between educators and learners [2].

\subsection{Interruption Factor on the Learning Process}

It is important to consider the interruption factor in the learning process. Interruptions are manifestations in a real-world context where multiple tasks are often taking place in parallel with the educator-learner interaction. The concept of interruption is relatively complex, and researching human interruption can be difficult. Interruption is a problem commonly encountered in educational institutions because educators and learners have cognitive limitations that restrict their abilities during the interaction, thereby resulting in temporary or full suspension of the learning process. These human limitations for handling interruptions may produce critical mistakes and affect the outcome of the learning interaction [3].

\subsection{Interruption Factor in Human-Computer Interaction (HCI)}

The interruption factor is not only present in the educator-learner interaction; interruption also emerges in $\mathrm{HCI}$ because humans have cognitive limitations affecting their performance, making them vulnerable to errors and delays in the interaction. In some situations, even very brief interruptions can have detrimental effects on human interaction. On the other hand, the computational expandability of interactive content may introduce greater applications that proactively push information towards the human; however, this information is not always appropriate, and incorrect information may increase a computer's potential to disrupt situations inappropriately [4]. The fact that the interruption factor may be continuous raises further questions: Can we keep the attention and interest of educators and learners during interactions using technology? How does the use of technology help to create solutions that support these interruptions?

\subsection{HCI Model Representation}

Interruption issues during the educator-learner interaction are immediate; this research proposes a model of interaction phenomena based on the learning process. The proposed model is a representation of HCI, where the learner is the human side and the educator is the computer.

In order to simulate the user and computer in the model, we use belief-desire-intention (BDI) agents: the user is the learner BDI agent (LA), and the educator is the educator BDI agent (EA); the LA simulates the learner's performance influenced by various factors (interaction level and distance), including emotional state, topic interest, and intentions. The EA monitors the learner's performance using situational perceptions to deliver adequate and appropriate content types, avoiding interruptions and keeping the interest of the learner in the context of the learning process interaction.

Our proposed model fits and supports the cognitive limitations of the learner; the learner will be able to perform various activities successfully at the time of the interaction.

\subsection{Degree of Engagement}

One objective of the EA is to keep a degree of engagement between educator and learner. A learner's participation or engagement levels vary in the interaction due to the degree of engagement; this is determined to be relative to the attentiveness or the interaction of a participant with the 
focus of attention. Some interruption factors contribute to an increase or decrease in the degree of engagement during the interaction; these include disturbances, noise, lack of feedback, prejudices, and level of interest in the content topic. Our model can represent the response in maintaining the degree of engagement [5].

\subsection{Model Validation}

In order to validate the model, we analysed the interactions of 500 users at an interactive museum in Tijuana, Mexico; the museum user was considered the learner and the exhibition as the educator. Data were gathered by observing user-exhibition interactions.

In order to achieve the above, we developed reactive modelling to maintain the frequent interaction in the learning process environment; we gave the learner a personalised environment (contents, services, information) to interact with his or her own interaction context. This research is also aided by different paradigms, such as multi-agent modelling to represent the involved elements in agents (learner and educator). We consider the behaviour of the learner's beliefs, desires, and intentions using the BDI paradigm. We use $\mathrm{HCI}$ to simulate the learner and educator interaction and Type-2 fuzzy logic to develop fuzzy perception in the educator BDI agent.

\section{Related Work}

Fuzzy logic has been seen to play an important role in situational perceptions research; prior research has been conducted on the reduction of interruption factors on HCI ([6], which introduces a fuzzy perception model for BDI agents to support the simulation of decision-making processes in environments with imperfect information; [7] introduced a graded BDI (g-BDI) agent development framework; g-BDI enabled the creation of agents as multi-context systems that provide the reason for three fundamental and graded mental attitudes (i.e., beliefs, desires, and intentions); [8] constructed an accurate model of the prevailing situation in order to make effective decisions about future courses of action within constraints using classical reasoning, augmented with a fuzzy component, generating beliefs in a fuzzy context of energy-awareness; [9] integrated the concepts of the BDI agent architecture into spatial issues; as a result, a novel spatial agent model was designed and implemented to analyse urban land use planning, the result of which showed the effects of spatial agents' behaviour, such as intention, commitment, and interaction on their decisions, always considering the uncertainty presented.

\subsection{Intelligent Environments}

The remarkable and rapid progress of research and technology allows us today to consider environments where the individual or group moves as a single entity able to understand the specific characteristics of a person or group to suit their needs and then respond intelligently to requests or appropriately respond in a natural and intuitive way. Of course, this environment must recognise the security and privacy needs of the individuals with respect to encountered situations. In the research field of intelligent environments (IEs), many scenarios can be provided as an example, such as a traveller who arrives at the airport in a foreign city where an IE program has been established; with this example system, the person is identified and verified immediately by the immigration authorities; they are given guidance on the rental of a car and a recommended route to their hotel. When the traveller arrives at the hotel, the room intelligently matches her/his personality with room features, including temperature, music, lighting, etc. IE may be seen as the coming together of different research areas [10], and it is seen to have some principal characteristics: ubiquity, context-awareness, natural interaction, and intelligence.

Perceiving the Learning Environment

A person's perspective can be described as the intention of a user to understand the situation that another user perceives [11]. As this definition highlights, the precise mechanisms of perspective are 
not highly specified, although research in cognitive psychology has suggested a shifting of attention to features of the environment that have less emphasis on an egocentric viewpoint [12,13].

The learning environment can be considered a place where learning is fostered and supported. For the learning environment to be fuzzy, it cannot be fully predefined. Sometimes, the users are involved in choosing learning activities and controlling the pace and direction of delivery, and accordingly, uncertainty and lack of control come into play. This place is the guide in the condition of continuing tentativeness and guardedness, but despite much care and attention, the system will often be chaotic to users. The complex nature of the learning environment interaction requires careful planning and design to avoid issues arising. An effective learning environment needs to be complemented by additional power resources of other users and the surrounding environment and culture [14].

\subsection{Computational Intelligence}

Computational intelligence (CI) is a well-established paradigm with current systems having defined computer characteristics that perform different tasks; sometimes, these tasks can be complicated and performed only by conventional actions. CI involves adaptive mechanisms to perceive and learn the intelligent behaviours presented in complex and chaotic environments; it also possesses attributes of abstraction, discovery, and association [15].

CI is a fast-moving, multidisciplinary field; it covers disciplines such as algorithms, data structures, neuro-computing, and artificial intelligence [16]. Nowadays, CI has attracted more attention over traditional artificial intelligence due to its tolerance of imprecise information, partial truth, and uncertainty [17]. Artificial intelligence is inefficient when solving problems with large input sizes (e.g., in data mining), whereas CI can support these.

\section{Fuzzy Inference Systems}

Computing using inference based on fuzzy logic is a popular method of computing, with many applications in areas such as control classification, expert systems, robotics, and pattern recognition adopting this method. The fuzzy inference system (FIS) is known by many names, such as the fuzzy expert system, the fuzzy model, fuzzy logic associative memory, and the fuzzy controller (also known as fuzzy rule-based systems). The FIS represents the major unit of a logic system. It can formulate adequate rules, based on which the decision is made. Figure 1 depicts the FIS structure.

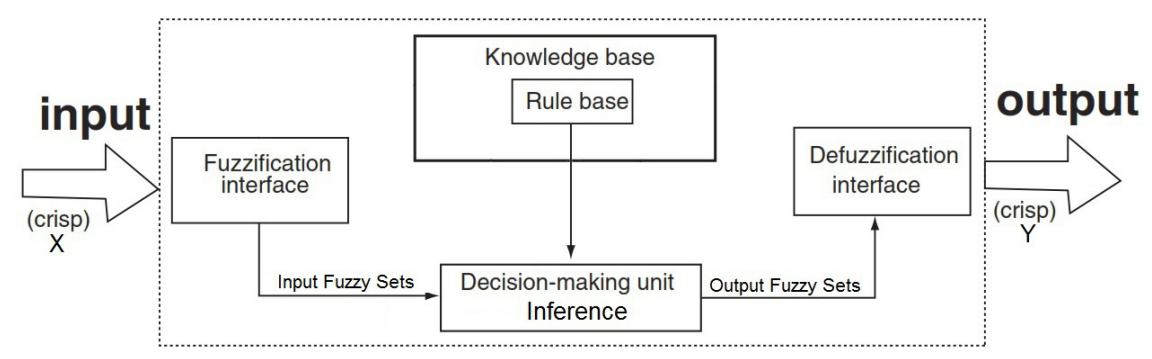

Figure 1. Fuzzy inference system.

An important element of the FIS is fuzzy sets, whereby knowledge is partitioned into particular situations; the fuzzy set is defined by its vague and ambiguous features, and limits may also be specified ambiguously. Crisp sets are those without ambiguity in their membership functions. The fuzzy set theory can deal powerfully with the presented ambiguity.

The work in $[18,19]$ proposed the concept of Type- 1 and Type- 2 fuzzy sets. Type- 1 fuzzy sets are described by membership functions that are aligned with numeric values between $[0,1]$, whereas Type- 2 fuzzy sets are described by membership functions that are themselves fuzzy, with linguistic and subjective values. Type- 1 fuzzy sets are certain. Type- 2 fuzzy sets are useful 
in scenarios where it is complicated to determine exact membership functions due to the uncertainty present; accordingly, they can be used to handle rule uncertainties and measure uncertainties. For these reasons, we chose to work with Type-2 FIS, because it allowed for the handling of arising uncertainties; additionally, the case study reported is a dynamic context where uncertainty exists all of the time and at every moment.

\subsection{Agent-Based Modelling}

Agent-based modelling (ABM) is increasingly applied to numerous empirical situations [20]. Its methodological advantage lies explicitly in its ability to simulate human decision-making processes while considering high degrees of heterogeneity [21,22]. ABM addresses the primary drawback of current simulation programs, which are limited in uncertainty prediction and provide a dynamic response to those uncertainties [23]. ABM takes into account the behaviours that emerge from the interactions of numerous autonomous agents [24]. It is capable of addressing the uncertainty of real-world actions using fuzzy logic techniques, rough sets, Bayesian networks, etc. [25].

An agent based in ABM can think and act like humans operating under autonomous control and perceive its environment autonomously, adapting to changes in order to achieve certain goals [26]. In making behavioural decisions, ABM outperforms simple reactive "if-then" rules by allowing agents to learn and change behaviours in response to their experiences [27]. Even at the simplest level, an $\mathrm{ABM}$ consists of agents and the relationships between them; there could be valuable findings of the system as a whole [28].

BDI Agent Architecture

Nowadays, new cooperative strategies for multi-agent systems and the combination of high-level compressed state representations and hybrid reward functions produce the best results in terms of task completion rates and learning efficiency. In the BDI paradigm, agents' states are represented through three types of components: beliefs, desires, and intentions. From the viewpoint of sociology and psychology, it has been an important direction using the BDI model to study agent modelling [29].

The BDI model is an abstraction of human deliberation based on rational actions theory in the human cognitive process [30]. Intention is subsequently planned and executed. A deliberation process selects the optimum goal from a set of possible options that meet a specific desire.

Goal-oriented approaches were advanced, as well as the requirements for the modelling method to assess likely user actions [31]; this incorporates a notion of the awareness of soft human behaviours into the system design and has been adopted by the goal-oriented requirements agents [32].

The BDI model has become almost a norm in the field of multi-agent systems (MASs) [33].

\section{Interactive Museum Case Study}

In order to validate the proposed model, we analyse and observe, identifying the involved elements during the learning process among educator and learner. The case study was carried out by modelling scenes on interactive environments that may represent a magnificent place for modelling interaction. In these environments, we find a variety of interactive exhibitions from which different situations emerge due to the presence of groups of people; otherwise, we find interruption factors causing incomplete interactions, reducing performance and increasing error rates, affecting user attention, as well as the emergence and variety of scenarios that can provide feedback to the research.

Due to its facilities and dynamism during daily activities, the Interactive Museum of "El Trompo", located in Tijuana, Mexico, was chosen as a suitable place for our study. This is because it is an interactive educational museum dedicated to youths. Its primary goal is to provide a place to interact and play while learning. 


\subsection{Methodology}

\subsubsection{Room Selection}

In order to analyse user-exhibition interaction in a better way, we studied (data sheets) the museum rooms, their themes, objectives, goals, methods of interaction, and their logistic location physically and theoretically; additionally, we observed the methods of interaction found in every room in order to select a suitable room that allowed us to analyse the behaviour, actions, performance, interruption factors, interaction distance, and interaction levels of users, as well as the interactive content type, information, and/or services of exhibitions provided. We also considered whether the content was adequate for users, suitable in relation to the kind of interactions of users, and adequate in maintaining the attention of the user. We further examined whether the content was harmful in causing interruptions or useful in avoiding interruptions. Additionally, we analysed the objective to determine whether it was adequate at encouraging a good interaction for the users and the media interface of the exhibition modules to determine whether they were adequate to have a good interaction.

\subsubsection{Exhibition Module Selection}

After analysis of the different exhibition modules, an interesting interactive module was chosen with features that allowed us to obtain the majority of the parameters to analyse in the research. The name of the exhibition module chosen was "Move Domain". The educational experience involved users interacting and playing with one of four objects (car, plane, bike, or balloon), which were displayed simultaneously on four separate screens, demonstrating the four different methods of moving in the simulated virtual world. Users were able to have the experience of using all four transportation means; they were able to interact in the virtual world and see how other users travel and interact around the virtual world. The exhibition's objective was to allow users to develop hand-eye coordination skills and spatial orientation using technology. The content was based on eye coordination and interaction with electronic games, with the exhibition's message being "I can learn about virtual reality through playing". The suggested number of users allowed at the same time was four.

\subsubsection{Exhibition Module Interface}

The module interface consisted of four sub-modules attached to connectors. Each module included a cover stand for the 32 inch screen, software that simulated the virtual world, and a cabinet to protect the computer.

The exhibition module was provided with a joystick to handle the plane, a steering wheel and pedals to drive the car, handlebars to ride the bike, and a rope to fly the balloon.

This interactive exhibition module is one of the most visited in the museum, and allowed us to obtain important data for analysis, processing, and validation of the model. Figure 2 depicts the analysed module.

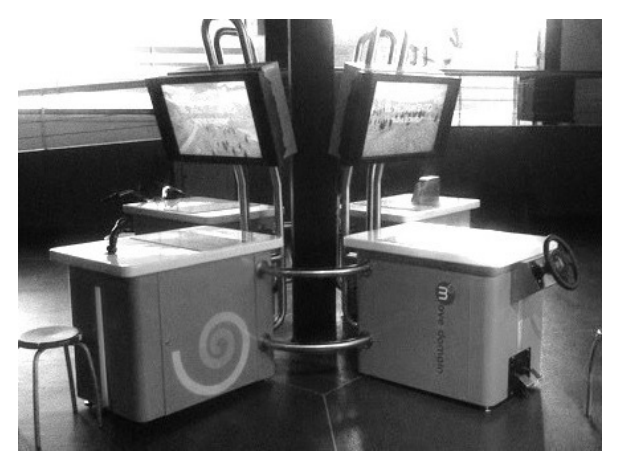

Figure 2. The analysed interactive exhibition module. The figure shows the analysed module; this is composed of four sub-modules with different user interfaces. 


\subsection{Study Subjects}

As subjects for the study, users were randomly selected from those children and adults who participated in supervised tours as a part of a permanent program of collaboration between local schools and the museum. The institution has the necessary agreements in place with the schools to conduct noninvasive interactive module evaluations to improve their design.

We evaluated user interaction-interruption behaviour by performing ethnographic research (notes style) to observe in a noninvasive way. Personal data were not required; therefore, information was produced directly in the museum room through real-time observations in line with institution committee recommendations to guarantee the anonymity of users.

Evaluation Interaction Parameters

We analysed and studied parameters such as interaction level (Which influencing factors increase or decrease the level of interaction? What is the quality of interaction? What is the interaction time? Which are the interaction abandonment factors?), presence (Do the users have a constant presence? Do the users have intermittent presence?), interactivity (Do the users have interactivity directly or indirectly with the exhibition? Do the users have shared interactivity with the exhibition?), control (Do the users have full control over the exhibition?), feedback (Do the users receive some feedback about the content?), creativity (Do the users change the way they interact according to their creativity?), productivity (Do the users propose something that changes the interaction?), communication (Do the users have communication directly from the exhibition?), adaptation (Do the users adapt their actions according to the interactive content type delivered by the exhibition?), and distance (is the users' distance adequate to interact with the exhibition? Is the distance a factor in interrupting or improving the interaction?).

All data collected were analysed in order to develop the FIS with all possible factors involved in order to obtain an adequate content interactive type. Figure 3 depicts in detail the average results of the parameters of the 500 users analysed.

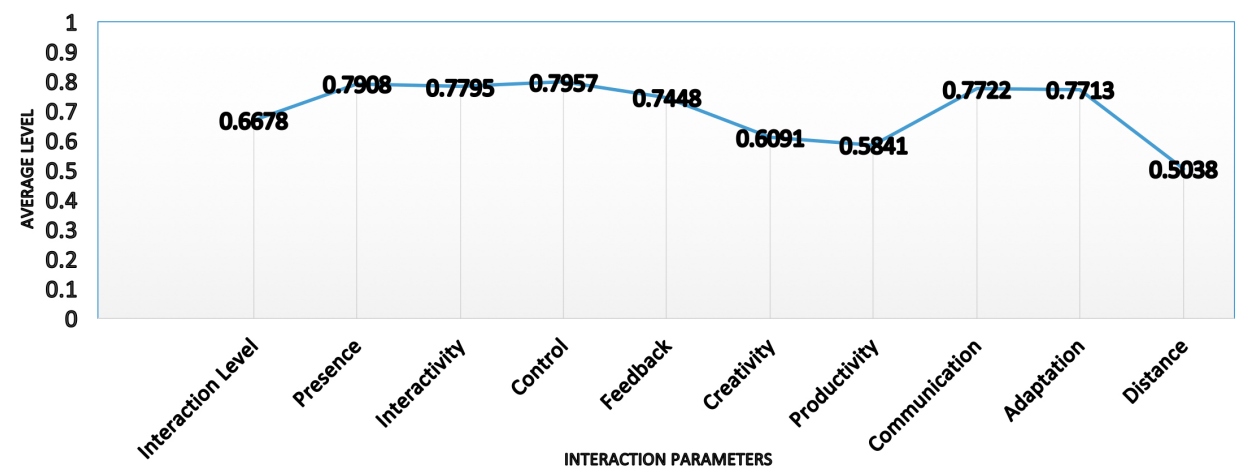

Figure 3. Average of interaction parameters. The figure shows the interaction parameters necessary to develop the adequate fuzzy inference system (FIS) in order to obtain the suitable interactive content type.

\section{Modelling Interaction on HCI}

To support user interactions, $\mathrm{HCI}$ is operating as a background process, using invisible sensing computational entities to interact with users. In our research, these entities are simulated by the learner BDI agent and the educator BDI agent. The entities' collaboration permits the $\mathrm{HCI}$ to deliver a customised interactive content type to users in a noninvasive manner which is context-aware.

The relationships between users (museum users) and computers (exhibition) need to be systematically modelled and represented to be ready for the emergent context; for this reason, we represent user-exhibition relationships. In addition, if we add tools such as JT2FIS [34] to facilitate 
these interactions, it should be possible to deal with the uncertainty presented by the inputs (interaction level, distance), task conflicts, and interruptions that occur when a user is involved in multiple interactions simultaneously.

Interactions-both personal and business related-are an essential part of our daily lives; these interactions are responsible for our well-being and productive work or business environment. The proliferation of mobile devices and pervasive computing technologies has radically changed the ways in which people communicate and the interaction between them. These devices support human interactions as they are equipped with software that helps users in coordinating, competing, and collaborating with each other [35].

In our research, we represent HCI simulated on a museum modelled with embedded agents (LA, EA) that allow the user-exhibition interaction to be supported. Our modelling proposal provides dynamic support for interactions, and it is aware not only of the user's physical context, but also of the social context (i.e., when a user interacts with another user). Our model consists of contextual attributes, such as the location of the user and what they are doing during the interactions. The social relationships are among users and other actors (teacher, museum guide, classmates, etc.) that influence an individual's action and performance during the interaction.

The idea for modelling the interaction on HCI in this research is important because it helps to improve the interaction level experience, offering an adequate interactive content type based on interaction level and distance to avoid possible interruption factors. Intensive research has been carried out in context modelling and context-awareness systems, focusing on physical contexts. However, these studies are not particularly explicit in addressing or modelling user-exhibition interactions and coordination functionality to improve the interaction quality experience.

Moreover, modelling the interaction on $\mathrm{HCI}$ provides opportunities to improve the interactions among the user and computer engaging in spontaneous activities as in our case study. Our HCI modelling selects and delivers adequate content and information to support the interactions of a large number of diverse users. Our proposed model supports spontaneous interactions and interruptions, coordinating available content during the course of the interaction.

Related work on supporting the interactions of users with available content in interactive environments has tended to focus on recognising activities based on the identification of meanings attached to a place. However, they do not account for the new requirement of spontaneity. A way to realize spontaneity is the approach of task-oriented computing [36].

\subsection{Representing HCI in a Museum}

Currently, museums are defined as non-profit institutions that serve to acquire, conserve, research, communicate, exhibit, and study educational material for the purpose of learning and customer enjoyment. Given the significant influence of museums on society, researchers have paid them much attention; for instance, Reference [37] studied the dynamic interaction between perceived quality and emotion as determinants of visitor satisfaction.

In our research, we modelled interactive museum elements; the museum user was represented by LA and the exhibition by the EA. In order to know the interactive content type offering adequate interactive information avoiding interruption, we used inputs like interaction level [38] and distance measuring [39]; the distance is randomly simulated, based on the measurement of the effective reading distance of passive tags embedded in the environment.

In the measurement of the distance and the level of interaction, some numerical results were obtained. These could be the inputs to our FIS. This practice measuring entails the following reflection: Does a metric allow us to properly measure the distance and interaction level of the user? For this reason, our model is composed of linguistic input variables with their respective membership functions to the interaction level (intlevel0, intlevel1, intlevel2, intlevel3, intlevel4, intlevel5) and distance (far, medium, near); linguistic variables can be considered a measure of the belief of the user (LA) and exhibition (EA). 
Within the existing model, the implementation of the BDI paradigm was chosen because the propositions of beliefs represent the level of interaction and distance where the BDI agent assumes the user has a specific evaluated interaction level and distance. Intentions are a subset of desires that should encourage and assist the user in the process of interaction through interactive activities. In order to approach the relevance to integrate fuzzy logic modelling to formalise a BDI agent's beliefs, recognising that transition ranges exist on interaction levels and distance, the use of fuzzy logic is deemed appropriate for exhibition (EA) reasoning, in order to deliver the adequate content type.

This research analyses data obtained from BDI agents (EA and LA) simulating the interaction among the user and exhibition. The educator BDI agent confronts its desires with its beliefs using fuzzy logic to infer relevant information on the interaction level and the distance of users' performance. This information is obtained using fuzzy perceptions.

\subsubsection{Modelling Museum Elements}

In our research, we define HCI as interactive museum elements composed of two principal actors: user (LA) and exhibition (EA). Through their performance (distance and interaction level), the user (LA) is evaluated by fuzzy perceptions of the exhibition (EA), obtaining (using the proposed FIS) the adequate interactive content type, ensuring the user's interest is maintained and avoiding the interruption factor. These agents have direct communication, constantly requesting and receiving information. The exhibition (EA) contains all information of the interactive exhibition it can collect through sensing using fuzzy perceptions; it can sense the performance data (interaction level and distance) of the users (LA). Interaction level and distance operate as feedback data to be processed by reasoning of FIS to offer the adequate interactive content type to help and increase the interaction quality experience of the user (LA), maintaining attention for the exhibition and avoiding the interruption factor.

The user (LA) and exhibition (EA) involved in the HCI receive and request all changes occurring during the interaction; consequently, the agents are then ready for emergent changes. This is of great importance, as it feedback information due to their awareness of the context at all times. Figure 4 represents the two agents involved in HCI.

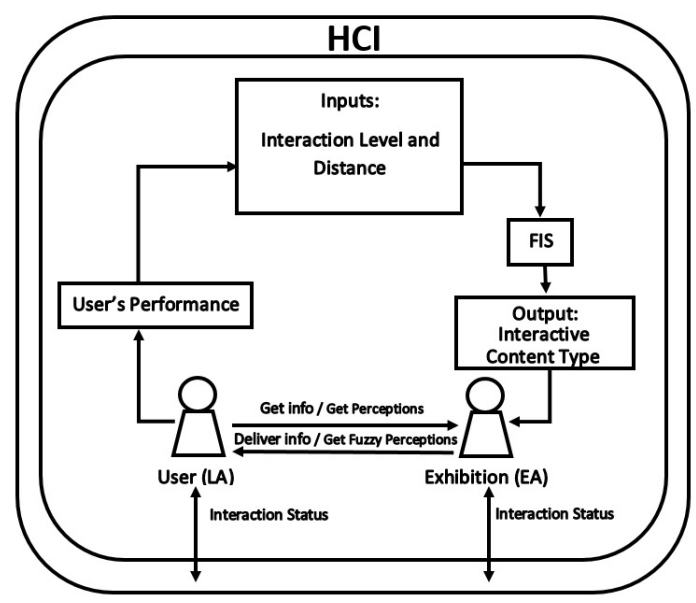

Figure 4. Agents involved in human-computer interaction (HCI). The figure shows the principal agents' interactions among each other and their context. LA, learner agent; EA, education agent.

The definition of the involved agents is based on the characteristics of the HCI. This means that the resources that provide services and information are based on user performance (distance, interaction level). The HCI serves as a mediator to update the agents with the status and changes that occur during the interaction. The EA is a representation of a BDI agent with mental states. The beliefs and desires of the agent involve definitions, estimating that the user has a certain distance and the level of 
interaction during a given period; this estimation can deal with uncertainty, determined by a set of membership functions.

The EA can perceive its interaction context with perceivers (fuzzy perceptions) and act accordingly with effectors (intentions). The exhibition (EA) perceivers must perceive and act to satisfy the user's requirements. Perception is an internal action where the agent captures information from its interaction context, formulating its beliefs from perceptions combined with prior knowledge of the interaction context (i.e., the EA has an interactivity perceiver that can measure the interaction level of a user to some degree of error; due to its prior knowledge, it creates the belief if the user has a high or low interaction level).

On the other hand, acting is the performance of an external action where the agent generates changes that modify the interaction context, offering adequate interactive content or customised services to the user. In this research, we developed powerful agents that covered the prior characteristics, implementing BDI paradigms such as such Jason [40], based on the AgentSpeak (L) introduced by Rao and Georgeff [41]. Figure 5 illustrates the process of the rationalization of the exhibition (educator BDI agent).

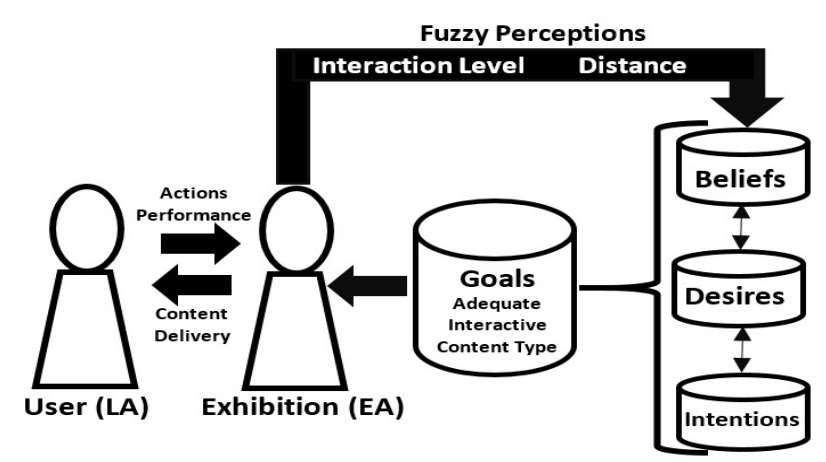

Figure 5. Exhibition (educator BDI agent) rationalization. The figure illustrates the rationalization process elements: the beliefs, desire, and intentions which are key to obtain the goals-in this case, to deliver the adequate interactive content.

The exhibition's belief base has several defined plans. Each plan is specified by different membership functions of linguistic variables that are received from the interaction, level, and distance in the process of the interaction. These plans have implications through beliefs: beliefs over desires and desires over intentions; consequently, this assists in determining the most appropriate interactive content type for the user, avoiding interruptions because it is based on the user's performance.

\subsubsection{Development of Museum Elements}

In our research, $\mathrm{HCI}$ is represented in an interactive museum. The involved elements-user (LA) and exhibition (EA) - are developed and simulated with a Jason-based programming language on AgentSpeak (L) ([40-43]).

The insertion of the proposed interaction context consists of hybrid BDI-fuzzy elements, binding the BDI and fuzzy logic paradigm. The idea here is to offer adequate interactive content types, avoiding interruptions that provoke incomplete interactions in the HCI.

The developed agents sometimes conduct simple tasks, performing only occasional database queries, sending data and information processed to responsible agents. Our reactive and involved agents-LA and EA-are in constant readiness to obtain information from the interaction context.

The communication among the agents is composed of four elements: receiver agent, sender or issuer agent, type of action, and message content. Messages can be sent and received to instances of LA and EA. The type of action defines which message will be sent (i.e., reporting the status of the HCI or informing about a user's performance). 
The message content has different types of information values, including interaction level (value among $0-5$ depending on the user interactivity), user-exhibition distance (value among near, medium, and high), interactive content type delivered (audio, graphic, text, and video; considering that audio content requires low interaction, graphics content requires medium interaction, text content requires high interaction, and video content requires extremely high interaction). Figure 6 depicts the communication process among agents.

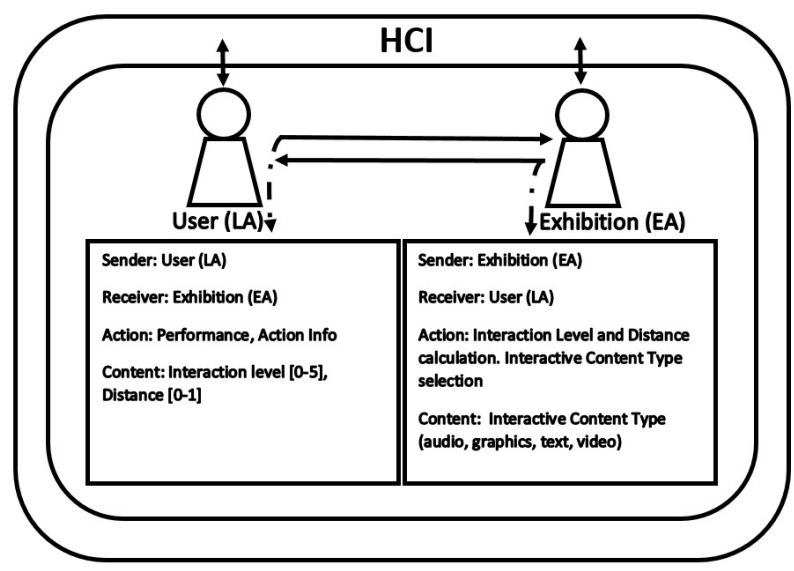

Figure 6. Communication process among agents. The figure shows the agent's communication process and is composed of four elements: sender, receiver, action, and content.

\subsubsection{Fuzzy Perceptions in the Museum Elements}

The logic in using BDI and the fuzzy logic paradigm in the museum elements is to help handle uncertain information in order to present adequate and appropriate interactive content. A belief is prepared in accordance with environmental inputs (interaction level and distance), with the respective membership functions. These variables act as inputs to the FIS, which define the output (interactive content type). The result of these beliefs is written by a fuzzy value and, in this case, given a linguistic value. The update process is dynamic and is altered according to the user's performance. The membership functions are modelled considering an initial belief based on fuzzy perceptions and ensuring an accurate result for assessing the user interaction.

The implementation of the FIS is solely for the purpose of effective utilization; this requires the use of programs that directly apply fuzzy logic functions. Some utility programs have specific modules to facilitate the accomplishment of this task and provide the necessary tools to conduct effective fuzzification; these include the utility JT2FIS [34], which is a Java class library for an interval Type-2 fuzzy inference system, used to build intelligent object-oriented applications. It also provides an effective fuzzification method and tools. The JT2FIS utility is used in this research.

The HCI inputs are the input variables that can be perceived by the EA. They are called the performance data of the user's interaction. On identifying the input variables (interaction level and distance) and the output variable (interactive content type) for the FIS, these are associated with a set of membership functions.

These functions comprise linguistic variables for the input "interaction" (intlevel0, intlevel1, intlevel2, intlevel3, intlevel4, intlevel5), for the input "distance" (far, medium, and near), and for the output "interactive content type" (audio, graphics, text, and video). Gaussian functions were used in all inputs and outputs because this type of membership function has a soft non-abrupt decay.

The FIS was implemented using the Mamdani method with the minimal implication operator and the defuzzification method of the centroid.

We defined 18-inference IF-THEN rules covering all linguistic variables; the rules are composed by the operator associated with the minimum method. Aggregation rules are made by the maximum 
method. The proposed FIS is flexible and permits the addition or deletion of rules; this can be seen as an advantage, as it can be adapted to different contexts or, if different variables exist, can be increased.

The FIS was also configured considering the performance of the 500 users. It is one of the most important elements of the proposed model, as it represents the inference that can be considered as the knowledge base. Table 1 shows the base rules (the knowledge base representation used in this research).

Table 1. Inference fuzzy rules of the FIS.

\begin{tabular}{ll}
\hline No & Inference Fuzzy Rules \\
\hline 1 & If (IntL is IntL5) and (Dist is Near) then (IntConType is video) \\
2 & If (IntL is IntL5) and (Dist is Med) then (IntConType is video) \\
3 & If (IntL is IntL5) and (Dist is Far) then (IntConType is text) \\
4 & If (IntL is IntL4) and (Dist is Near) then (IntConType is video) \\
5 & If (IntL is IntL4) and (Dist is Med) then (IntConType is video) \\
6 & If (IntL is IntL4) and (Dist is Far) then (IntConType is text) \\
7 & If (IntL is IntL3) and (Dist is Near) then (IntConType is video) \\
8 & If (IntL is IntL3) and (Dist is Med) then (IntConType is text) \\
9 & If (IntL is IntL3) and (Dist is Far) then (IntConType is grfs) \\
10 & If (IntL is IntL2) and (Dist is Near) then (IntConType is text) \\
11 & If (IntL is IntL2) and (Dist is Med then (IntConType is text) \\
12 & If (IntL is IntL2) and (Dist is Far) then (IntConType is audio) \\
13 & If (IntL is IntL1) and (Dist is Near) then (IntConType is grfs) \\
14 & If (IntL is IntL1) and (Dist is Med) then (IntConType is grfs) \\
15 & If (IntL is IntL1) and (Dist is Far) then (IntConType is audio) \\
16 & If (IntL is IntL0) and (Dist is Near) then (IntConType is grfs) \\
17 & If (IntL is IntL0) and (Dist is Med) then (IntConType is audio) \\
18 & If (IntL is IntL0) and (Dist is Far) then (IntConType is audio) \\
\hline
\end{tabular}

\subsubsection{Fuzzy Perceptions Process}

An important process that impacts all museum elements is how the HCI-aided by exhibition (EA)—obtains fuzzy perceptions from the user's performance. This process begins with the perceiver $(\chi)$ observation of changes in the surrounding environment; this change can be represented as a set of indicators $(\zeta)$, with every indicator being described by set membership function values $(\pi)$. The indicators $(\zeta)$ can be perceived by perceivers $\chi$, which can sense these values and consider the inputs (interaction level and distance) of the FIS. Through its inference, the FIS generates the output (interactive content type). The fuzzy value result will be considered a belief atom $\kappa(\tau)$, where $\kappa(\tau) \varepsilon \Omega$ is a belief set. Figure 7 depicts the fuzzy perception process of the exhibition EA.

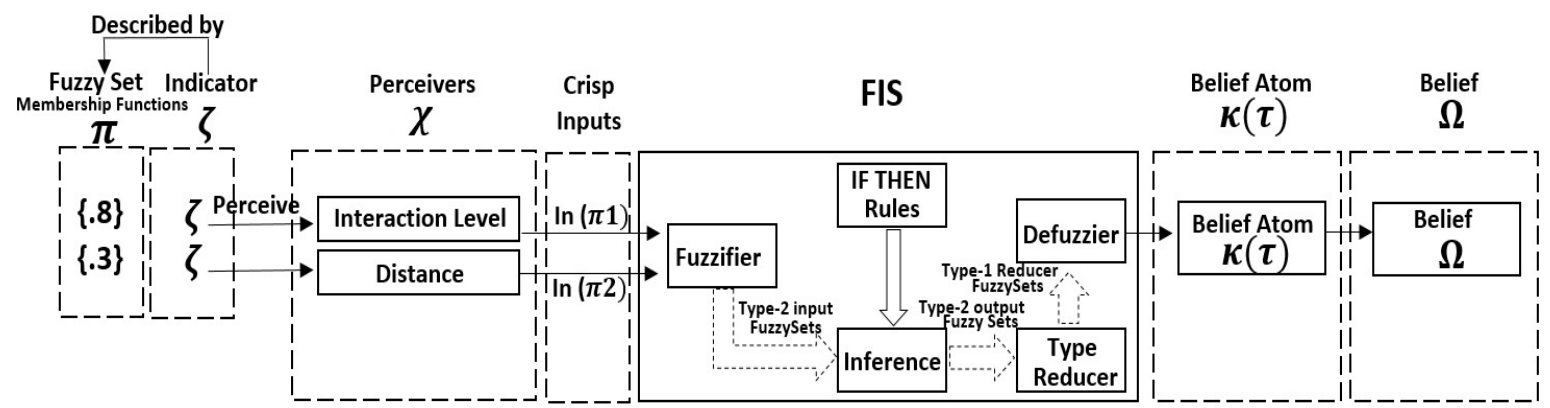

Figure 7. Fuzzy perception process. The figure shows the fuzzy perception process. This process is the key to creating the fuzzy perceptions of the EA in order to deliver the adequate interactive content type, considering the user's performance (interaction level and distance). 


\subsubsection{Illustrative Example}

The following example illustrates how the entities in this case (museum visitor, represented by LA) can change their performance (interaction level and distance) during a museum tour day and how the exhibition can perceive the performance changes using fuzzy perceptions.

Let us consider that a visitor takes a museum tour. At the beginning of the tour, the visitor starts the interaction on the first exhibition modules; at this point, the visitor's performance is interaction level is " 5 " and distance is "near" the exhibition (let us suppose the visitor has much energy and interest in the exhibition).

Therefore, the EA, using fuzzy perceptions, implies its beliefs, then based on its beliefs, the resultant belief is a video (fuzzy value) because it perceives high interaction (0.9) and near distance (0.9). Later, let us suppose that the visitor is in the middle of their museum tour (the visitor decreases their performance and interest). Now, the interaction level is " 2.5 ", and the distance is "medium" with respect to the exhibition. Therefore, the EA, using fuzzy perceptions, implies its beliefs, then based on its beliefs, the resultant belief is graphics (fuzzy value), because it perceives medium interaction (0.4) and medium distance (0.4). Finally, let us suppose that the visitor is at the final part of the museum tour (the visitor's performance and interest continues to decrease during their visit). Now, the interaction level is " 0.5 ", and the distance is "far" from the exhibition. Therefore, the EA, using fuzzy perceptions, implies its beliefs, then based on its beliefs, the resultant belief is audio (fuzzy value) because it perceives low interaction (0.1) and distance far (0.1).

Table 2 depicts the exhibition (domain) agent's mind state, elements, interaction level, and distance presented.

Table 2. Mind state values of exhibition EA.

\begin{tabular}{ccclc}
\hline Interaction Level & Distance & Element & Description & Source \\
\hline 5 & Near & Belief & video(fuzzy value(.9),(.9)) & percept \\
2.5 & Medium & Belief & graphics(fuzzy value(.4),(.4)) & percept \\
0.5 & Far & Belief & audio(fuzzy value(.1),(.1)) & percept \\
\hline 5 & Near & Events & + !select(video) & self \\
2.5 & Medium & Events & + !select(graphics) & self \\
0.5 & Far & Events & + +select(audio) & self \\
\hline 5 & Near & Intentions & + !deliver(video) & self \\
2.5 & Medium & Intentions & + !deliver(graphics) & self \\
0.5 & Far & Intentions & + +deliver(audio) & self \\
\hline
\end{tabular}

\section{Results}

In this section, the results obtained from the sample of 500 users visiting the interactive museum "El Trompo" in Tijuana, Mexico are presented and analysed. The users were evaluated and processed using a custom fuzzy c-means method of data mining named Data Mined Type-2 (DMT2F) [38].

\subsection{FIS Configuration}

Once all data were mined with DMT2F and 18 inference rules were added to build the knowledge base, we obtained as a result of the FIS configuration parameters that the FIS was configured with two inputs (interaction level and distance). These inputs were composed with the exact parameters considering the performance of the 500 users. These inputs are essential for feedback to the FIS; also, the FIS was configured with one output (interactive content type). This output is important because it delivers the adequate interactive content type in order to avoid interruption during the interaction.

Additionally, DMT2F considers the uncertainty of each user. This uncertainty is assigned automatically by the evaluation method of the FIS Type-2. The implementation of DMT2F is adequate 
in the interaction context because it is in context where uncertainty is contemplated and present at all times. During this process, an FIS with higher accuracy was obtained in the realized configuration, as we considered and undermined all possible input variables; consequently, an accurate interactive content type result was obtained during real-time interaction, improving the user's interest, attention, and interaction, and avoiding interruptions. Table 3 depicts the configuration parameters of the data mined Type-2 FIS.

Table 3. Inputs, outputs data mined Type-2 FIS configuration.

\begin{tabular}{|c|c|c|}
\hline Type & Member Function & Params (Values) \\
\hline $\begin{array}{c}\text { Input1 } \\
\text { (IntLevel) }\end{array}$ & $\begin{array}{c}\text { GaussUncertainty } \\
\text { MeanMemberFunction }\end{array}$ & $\begin{aligned} \text { IntLevel0 } & =\left[\begin{array}{lll}0.486 & 1.709 & 2.196\end{array}\right] \\
\text { IntLevel1 } & =\left[\begin{array}{lll}0.373 & 2.387 & 2.761\end{array}\right] \\
\text { IntLevel2 } & =\left[\begin{array}{lll}0.300 & 3.021 & 3.321\end{array}\right] \\
\text { IntLevel3 } & =\left[\begin{array}{lll}0.284 & 3.607 & 3.891\end{array}\right] \\
\text { IntLevel4 } & =\left[\begin{array}{lll}0.286 & 3.676 & 3.963\end{array}\right] \\
\text { IntLevel5 } & =\left[\begin{array}{lll}0.376 & 4.132 & 4.508\end{array}\right]\end{aligned}$ \\
\hline $\begin{array}{c}\text { Input2 } \\
\text { (Distance) }\end{array}$ & $\begin{array}{c}\text { GaussUncertainty } \\
\text { MeanMemberFunction }\end{array}$ & $\begin{array}{l}\text { Far }=\left[\begin{array}{lll}0.196 & 0.312 & 0.509\end{array}\right] \\
\text { Medium }=\left[\begin{array}{lll}0.200 & 0.372 & 0.573\end{array}\right] \\
\text { Near }=\left[\begin{array}{lll}0.217 & 0.492 & 0.71\end{array}\right]\end{array}$ \\
\hline $\begin{array}{c}\text { Output } \\
\text { (IntConType) }\end{array}$ & $\begin{array}{c}\text { GaussUncertainty } \\
\text { MeanMemberFunction }\end{array}$ & $\begin{array}{l}\text { IntCnTypAud }=\left[\begin{array}{lll}0.090 & 0.463 & 0.554\end{array}\right] \\
\text { IntCnTypGph }=\left[\begin{array}{lll}0.066 & 0.597 & 0.663\end{array}\right] \\
\text { IntCnTypTxt }=\left[\begin{array}{lll}0.070 & 0.618 & 0.689\end{array}\right] \\
\text { IntCnTypVid }=\left[\begin{array}{lll}0.088 & 0.792 & 0.880\end{array}\right]\end{array}$ \\
\hline
\end{tabular}

\subsection{Interactive Content Type Results}

After using the DMT2F in our FIS, an evaluation was conducted to obtain adequate interactive content type results for the sample of 500 users. Table 4 depicts these analysed users.

Table 4. Results of the interactive content type using the data mined Type-2 fuzzy method (DMT2F).

\begin{tabular}{cccc}
\hline Subject & Interaction Level & Distance & Interactive Content Type Using DMT2F \\
\hline 1 & 1.6738 & 0.4168 & 0.5553 (audio) \\
2 & 2.0087 & 0.3130 & 0.0 .5451 (audio) \\
3 & 3.0073 & 0.6225 & 0.5921 (graphics) \\
4 & 4.1161 & 0.8769 & 0.7665 (video) \\
5 & 4.3935 & 0.3055 & 0.7370 (video) \\
6 & 3.8101 & 0.3505 & 0.7166 (text) \\
$\ldots$ & $\ldots$ & $\ldots$ & 0.7679 (video) \\
500 & 4.3870 & 0.6969 & \\
\hline
\end{tabular}

Interactive Content Type Percentage

In this research, we obtained noteworthy results about the percentage of interactive content types delivered. The sample results obtained were: $20 \%$ of users received content type "video"; $21 \%$ of users received content type "audio"; $32 \%$ of users received content type "text"; and 27\% of users received content type "graphics". This means that the interactive content type "text" is the most adequate to interact with in this kind of interactive environment, helping to avoid possible interruptions originating from inadequate content. Figure 8 depicts these results. 


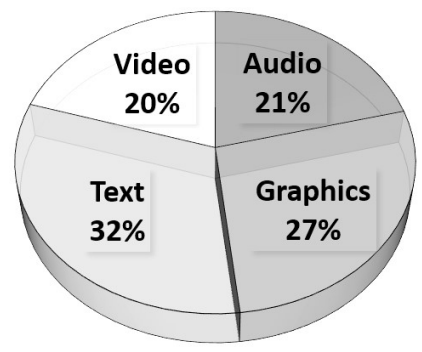

Figure 8. Interactive content type percentage. The figure shows the interactive content type percentage of the 500 users analysed.

\section{Discussion}

Nowadays, technology is being increasingly used in museums around the world. However, in Tijuana, Mexico, not many museums are adopting new technologies, with many simply offering the same content type in all of their exhibitions. On the other hand, we identify some museums, such as our case study "El Trompo" Interactive Museum in Mexico, which has adopted interactive exhibitions through the use of technology.

\subsection{Technology Use}

The use of technology has been seen to help attract more visitors to museums, with visitors demanding adequate and appropriate interactive content types that permit and encourage their interest throughout the museum, avoiding interruptions that impact interaction. One way to achieve this is through the proposed model, which offers museum visitors experiences that directly impact their knowledge and interest. Our model evaluates and perceives user uncertainty in their interaction (level and distance), aided by fuzzy perceptions in EA. After this perception, the output interactive content type is identified, offering suitable services and information; having an impact and improving interest avoids interruptions or abandonment of the museum's exhibitions.

\subsection{Applying Fuzzy Logic}

Our agents-particularly the domain (EA) with mental states-can perceive interruptions at the moment of the first interaction through the recognition of the user's performance (interaction level and distance). The use of fuzzy logic to reconstruct the beliefs on BDI EA can deal with uncertainty in a model built in part from mental states. This integration allows the analysis of uncertainty in the user's performance process, resulting in a suitable profile of each user's performance.

\subsection{Interruption Factor}

The user's low performance is a consequence of interruption factors during the interaction, and in some cases, it takes time to recover from or could represent a complete interruption of interaction. In order to identify how to avoid interruptions, some questions arise: How can we recover from irregular interruptions, or how we can avoid interruptions? The response is through the proposed research where we analysed factors and offered adequate interactive content types, which can avoid these interruptions. If we deliver the adequate and appropriate content, we can help users recover from suspended interaction.

\section{Interruption Factor Recovery}

When a user is interrupted, it is necessary to recall their progress before the interruption occurred, but if we are able to recall this progress with the adequate content, the interruption's recovery is much faster; otherwise, the interruption's recovery can be slower. We can compare the proposed research with other recovery techniques [44,45], analysing ways to resume after interruption; through 
comparison, we can see that our research improves and strengthens the interaction in order to increase attention in the exhibition. Moreover, we analysed if negative effects of the interruption are avoided if the user's performance increases having a complete and uninterrupted interaction. Comparing our research with other research [46,47] that used avoidance of interruption techniques, none were seen to use fuzzy perceptions and did not consider users' uncertainty, which increases the error degree to avoid interruption.

\subsection{Performance of Users}

The user evaluation during $\mathrm{HCI}$ is completed to determine the adequate interactive content type based on performance, the gathering of relevant effects of interruptions, and the identification of what happens when users are interrupted by a particular interruption factor. This could include interruptions with content related to the exhibition, where the performance could be maintained, reduced, or minimised, but not representing the interaction. Otherwise, for interruptions that are not content related, the performance may degrade until null performance, representing an interaction abortion. If we deliver adequate content using our proposed model, it is important to resume interaction after interruptions. Understanding such adaptations will improve interaction level experience.

\subsection{Noninvasive Evaluation}

The sample of users was examined during their natural interaction experience. The proposed evaluation method is noninvasive because it operates in background mode. The users can interact naturally, obtaining real information in a natural way, influencing the quality of results.

\section{Conclusions}

During the learning process, we can identify many interruption factors. The proposed model helps the learner experience a quality means of learning, which is provided by the educator with all of the adequate interactive content types, improving performance and creating an effective learning process.

We have explored the idea that HCI enhancement-with adequate content types that avoid interruptions-could intensify the positive influence on learning and improve the relationship with other users; this can be beneficial to elevate the interactive level experience, allowing a direct repercussion in the quality of $\mathrm{HCI}$.

Through experimental research, we model and represent with agents the learner (LA)-educator (EA) interaction; both agents are provided with the BDI approach, which permits the creation of beliefs, desires, and intentions, allowing mental states for reasoning. The EA uses the fuzzy logic approach to have a fuzzy perception of the user's performance, improving interaction and avoiding interruptions.

We have demonstrated that if adequate and appropriate content types are offered, the interaction can be completed inclusively with some interruptions. We analysed if the content of the interruption is similar to the interaction context, identifying that not all interruptions can be deemed negative, with some interruptions helping users to complete their interaction. Otherwise, if the interruption content is completely different, it can increase the interruption level until the user's interaction is abandoned.

This research can act as an alternative for HCI researchers to approach successful interaction; it can represent an option to understand how to minimize or avoid the interruption factors during interactions.

Acknowledgments: We would like to thank The National Council for Science and Technology of Mexico, the Autonomous University of Baja California and the Interactive Museum 'El Trompo' for the support granted during this research.

Author Contributions: All of the authors conceived of and designed the study. Manuel Castañón-Puga and Felipe Lara-Rosano provided methodological advice. Ricardo Rosales, Nora Osuna-Millan and Maria Virginia 
Flores-Ortiz performed the field survey and analysed the data. Ricardo Rosales and Richard David Evans wrote the paper. All authors read and approved the manuscript.

Conflicts of Interest: The authors declare no conflict of interest.

\section{References}

1. Morley, D.; Parker, C. Understanding Computers: Today and Tomorrow; Cengage Learning: Boston, MA, USA, 2013.

2. Kolb, D.A. Experiential Learning: Experience as the Source of Learning and Development; Prentice-Hall: Upper Saddle River, NJ, USA, 1984.

3. Maureen, A.C.; Marsh, R. Interest level improves learning but does not moderate the effects of interruptions: An experiment using simultaneous multitasking. Learn. Individ. Differ. 2014, 30, 112-117.

4. McFarlane, D.C.; Latorella, K. Modifying the Classroom Environment to Increase Engagement and Decrease Disruption with Students Who Are Deaf or Hard of Hearing. J. Deaf Stud. Deaf Educ. 2012, 17, 518-533.

5. Guardino, C.; Antia, S. The Scope and Importance of Human Interruption in Human-Computer Interaction Design. Hum.-Comput. Interact. 2002, 17, 1-61.

6. Farias, G.; Dimuro, G.; Costa, A. BDI agents with fuzzy perception for simulating decision making in environments with imperfect information. In Proceedings of the Multi-Agent Logics, Languages, and Organisations Federated Workshops, Lyon, France, 30 August-2 September 2010; Volume 627, $23-41$.

7. Casali, A.; Godo, L.; Sierra, C. A graded BDI agent model to represent and reason about preferences. Artif. Intell. 2011, 175, 1468-1478.

8. Song, S.; O'Hare, G.; O'Grady, M. Fuzzy decision making through energy-aware and utility agents within wireless sensor networks. Artif. Intell. Rev. 2007, 27, 165-187.

9. Behzadi, S.; Alesheikh, A.; Sierra, C. Introducing a novel model of belief-desire-intention agent for urban land use planning. Eng. Appl. Artif. Intell. 2013, 26, 2028-2044.

10. Weiser, M. The computer for the 21st century. In Human Computer Interaction: Toward the Year 2000, 2nd ed.; Morgan Kaufmann Publishers: Burlington, MA, USA, 1995.

11. Tomasello, M.; Kruger, A.; Ratner, H. Cultural learning. Behav. Brain Sci. 1993, 16, 495-552.

12. Frischen, A.; Loach, D.; Tipper, S. Seeing the world through another person's eyes: Simulating selective attention via action observation. Cognition 2009, 111, 212-218.

13. Tversky, B.; Hard, B. Embodied and disembodied cognition: Spatial perspective-taking. Cognition 2009, 110, 124-129.

14. Gayle, W. Constructivist Learning Environments: Case Studies in Instructional Design; Educational Technology Publication Inc.: Washington, DC, USA, 1996.

15. Hassaniena, A.; Al-Shammari, E.; Ghali, N. Computational intelligence techniques in bioinformatics. Comput. Biol. Chem. 2013, 47, 37-47.

16. Konar, A. Computational Intelligence Principles, Techniques and Applications; Springer: Berlin/Heidelberg, Germany, 2005.

17. Andina, D.; Pham, D. Computational Intelligence for Engineering and Manufacturing; Springer: Berlin/Heidelberg, Germany, 2007.

18. Zadeh, L. Fuzzy sets. Inf. Control 1965, 8, 338-353.

19. Zadeh, L. The concept of a linguistic variable and its application to approximate reasoning. Inf. Sci. 1975, 8, 199-249.

20. Smajgl, A. Challenging beliefs through multi-level participatory modelling in Indonesia. Environ. Model. Softw. 2010, 25, 1470-1476.

21. Gilbert, N. Agent-Based Models; SAGE Publications: Thousand Oaks, CA, USA, 2008.

22. Parker, D.; Manson, M.; Janssen, M.; Hoffmann, M.J.; Deadman, P. Multiagent system models for the simulation of land-use and land-cover change: A review. Ann. Assoc. Am. Geogr. 2003, 93, 314-337.

23. Lee, Y.; Malkawi, A. Simulating human behavior: An agent-based modeling approach. In Proceedings of the 13th IBPSA Conference, Chambery, France, 26-28 August 2013.

24. Sokolowski, J.; Banks, C. Principles of Modeling and Simulation: A Multidisciplinary Approach; John Wiley and Sons: Hoboken, NJ, USA, 2009. 
25. Ramos, A.; Augusto, J.; Shapiro, D. Ambient intelligence-the next step for artificial intelligence. IEEE Intell. Syst. 2008, 23, 15-18.

26. Russell, S.; Norvig, P. Artificial Intelligence; A Modern Approach, 3rd ed.; Pearson Education International: London, UK, 2009.

27. Macal, C.; North, M. Tutorial on agent-based modelling and simulation. J. Simul. 2010, 4, 151-162.

28. Bonabeau, E. Agent-based modeling: methods and techniques for simulating human systems. Proc. Nat. Acad. Sci. USA 2002, 99, 7280-7287.

29. Dongning, L; Yong, T. Intelligent agents belief temporal substructure logic model. Comput. Appl. 2010, 27, 2448-2451.

30. Bratman, M. Intentions, Plans, and Practical Reason, 1st ed.; Center for the Study of Language and Information: Stanford, CA, USA, 1999.

31. Yu, E. Modelling Strategic Relationships for Process Reengineering, Social Modeling for Requirements Engineering; MIT Press: Cambridge, MA, USA, 2011.

32. Jian, Y.; Li, T.; Liu, L.; Yu, E. Goal-oriented requirements modelling for running systems. In Proceedings of the First International Workshop on Requirements@Runtime, Sydney, Australia, 28-28 September 2010; Volume 1, pp. 1-8.

33. Wooldridge, M.; Jennings, N. Intelligent Agents: Theory and Practice. Knowl. Eng. Rev. 1995, 10, 115-152.

34. Castañón-Puga, M.; Castro, J.R.; Flores-Parra, J.M.; Gaxiola-Pacheco, C.G.; Martínez-Méndez, L.-G.; Palafox-Maestre, L.E. JT2FIS A Java Type-2 Fuzzy Inference Systems Class Library for Building Object-Oriented Intelligent Applications. Adv. Soft Comput. Appl. Lecture Notes Comput. Sci. 2013, 8266, 204-215.

35. Castelfranchi, C. Modelling social action for AI agents. Artif. Intell. 1998, 103, 157-182.

36. Wang, Z.; Garlan, D. Task-Driven Computing; Carnegie Mellon University: Pittsburgh, PA, USA, 2000.

37. De Rojas, C.; Camarero, C. Visitors' experience, mood and satisfaction in a heritage context: Evidence from an interpretation center. Tour. Manag. 2008, 29, 525-537.

38. Rosales, R.; Flores, D.; Palafox, L. Representation of interaction levels using data mining and Type-2 fuzzy inference system. Front. Comput. Sci. 2017, under review.

39. Luh, Y.-P.; Liu, Y.-C. Measurement of Effective Reading Distance of UHF RFID Passive Tags. Mod. Mech. Eng. 2013, 13, 115-120.

40. Bordini, R.; Bazzan, A.; Jannone, R.; Bassao, D.M.; Vicari, R.M.; Lesser, V.R. Agentspeak: Efficient intention selection in BDI agents via decision-theoretic task scheduling. In Proceedings of the first international joint conference on autonomous agents and multi-agent systems, Bologna, Italy, 15-19 July 2002; pp. 1294-1302.

41. Rao, A.; Georgeff, M. AgentSpeak(L): BDI agents speak out in a logical computable language. In Proceedings of the Seventh Workshop on Modelling Autonomous Agents in a Multi-Agent World, Einhoven, The Netherlands, 22-25 January 1996; Volume 1038, pp. 42-55.

42. Moreira, A.; Vieira, R.; Bordini, R. Extending the operational semantics of a BDI agent-oriented programming language for introducing speech-act based communication. In Proceedings of the International Workshop on Declarative Agent Languages and Technologies, New York, NY, USA, 19 July 2004; pp. 135-154.

43. Bordini, R.; Hubner, J. Jason: A Java-based AgentSpeak interpreter used with saci for multiagent distribution over the net. In Proceedings of the 6th International Workshop CLIMA VI, London, UK, 27-29 June 2005.

44. Altmann, E.; Trafton, J. Memory for goals: an activation based model. Cognit. Sci. 2002, 26, 39-83.

45. Brumby, D.; Cox, A.; Back, J.; Gould, S.J. Recovering from an interruption: Investigating speed-accuracy tradeoffs in task resumption strategy. J. Exp. Psychol.: Appl. 2013, 19, 95-107.

46. Mark, G.; Voida, S.; Cardello, A. A pace not dictated by electrons: an empirical study of work without email. In Proceedings of the Conference on Human Factors in Computing Systems, Austin, TX, USA, 5-10 May 2012; pp. 555-564.

47. Relihan, E.; O'Brien, V.; O'Hara, S.; Silke, B. The impact of a set of interventions to reduce interruptions and distractions to nurses during medication administration. Qual. Saf. Health Care 2010, 19, 1-6.

(C) 2017 by the authors. Licensee MDPI, Basel, Switzerland. This article is an open access article distributed under the terms and conditions of the Creative Commons Attribution (CC BY) license (http:/ / creativecommons.org/licenses/by/4.0/). 